Proof. If we define the polynomials $h_{i}(z)$ recursively as follows: $h_{1}(z) \equiv f_{1}(z)$, and for $2 \leqq i \leqq k, h_{i}(z) \equiv f_{i}(z)+\left(\lambda_{i-1} / \lambda_{i}\right) h_{i-1}(z)$, we see that $F\left(z ; \lambda_{1}, \cdots, \lambda_{k}\right) \equiv \lambda_{k} h_{k}(z)$. Thus $h_{i}(z)$ is a linear combination of two polynomials to which Theorem 4 is applicable and the truth of Theorem 6 follows by induction.

\title{
REFERENCES
}

1. M. Marden, The geometry of the zeros of a polynomial in a complex variable, Math. Surveys No. 3, Amer. Math. Soc., Providence, R. I., 1949.

2. A. Ostrowski, Recherches sur la methode de Graeffe et les zeros des polynomes et des series de Laurent, Acta Math. 72 (1940), 99-257.

3. Z. Rubinstein, Analytic methods in the study of zeros of polynomials, Pacific J. Math. 13 (1963), 237-249.

4. W. Specht, Algebraische Gleichungen mit reelen oder komplexen Koeffizienten, Enzyklop. d. math. Wissen., 2nd ed., I 1, 3, II, Stuttgart, 1958.

5. J. L. Walsh, On the location of roots of certain types of polynomials, Trans. Amer. Math. Soc. 24 (1922), 163-180.

University OF MaryLand

\section{ZEROS OF EXPONENTIAL SUMS}

\author{
D. G. DICKSON
}

1. Introduction. This paper deals with the distribution in the complex plane of the zeros of an exponential sum of the form

$$
f(z)=\sum_{j=1}^{n} A_{j} z^{m_{j}}\left[1+\epsilon_{j}(z)\right] e^{\omega_{j} z},
$$

where $n>1$; the $A_{j}$ and the $\omega_{j}$ are complex numbers such that $A_{j} \neq 0$ and the $\omega_{j}$ are distinct; the $m_{j}$ are non-negative integers; the functions $\epsilon_{j}$ are analytic for $|z| \geqq r_{0} \geqq 0$ with $\lim _{z \rightarrow \infty} \epsilon_{j}(z)=0$. A function of the form

$$
\sum_{j=1}^{n} P_{j}(z) e^{\omega j z}
$$

Received by the editors September 9, 1963.

1 This work was supported by a grant from the National Science Foundation. 
where the $P_{j}$ are polynomials, is an important special case of (1).

When the $P_{j}$ in (2) are constants and the $\omega_{j}$ are real, the zeros of (2) are confined to a vertical strip in the plane. C. E. Wilder [8] found an estimate of the number of zeros in a rectangle with vertical sides on the boundary of such a strip. A strengthening by Langer [3] of Wilder's result showed that the number of zeros in such a rectangle of height $s$ with a zero-free boundary differs from max $\left|\omega_{i}-\omega_{j}\right| s /(2 \pi)$ by at most $n-1$. Allowing the $\omega_{j}$ to be complex, Tamarkin [7] found similar results for rectangles in strips in the direction of the exterior normals to the sides of the polygonal convex hull $Q$ of the set $\left\{\bar{\omega}_{j}\right\}_{j=1}^{n}$. These results together with the results of Pólya [4] and Schwengeler [6], which located the zeros of (2) of large modulus in strips that are logarithmic to the normals, were combined by Langer to obtain similar asymptotic results for zeros of (1) in sets that are asymptotically rectangular.

Our purpose here is to obtain results in which this asymptotic similarity is made explicit. Some results in this direction were obtained by the author in [1]. However, those results were limited in generality (in particular, regarding the dependence of parameters), having been designed for a limited application. An application of the more general results obtained here will be made elsewhere [2] to establish Pólya's remark [5, p. 549] to the effect that the number of zeros of (2) of modulus at most $r$ equals $L r /(2 \pi)+O(1)$, where $L$ is the perimeter of $Q$. This is a special case of a similar result for (1).

We will rely rather heavily on the notation and results in the early part of [1]. The notation will be described briefly in $\$ 2$. In $\$ 3$ a very slight strengthening of Langer's proof of Wilder's theorem will yield a best possible result. This will then be used to prove the main result given in Theorem 2 .

2. Preliminaries. We will use the generic symbol $\epsilon(z)$ to denote a function which is analytic for large $|z|$ in a region $R$ and which has the property that $\epsilon(z) \rightarrow 0$ as $z \rightarrow \infty$ in $R$. For convenience, by the zeros of $f$ we will mean the zeros of $f$ of modulus greater than $r_{0}$.

ln [1] the indices on the $\omega_{j}$ of (1) are labeled so that $\bar{\omega}_{k}$, for $k=1, \cdots, \sigma$, are the vertices of $Q$ given in a specific counterclockwise order. $L_{k}$ is the line segment $\left[\bar{\omega}_{k}, \bar{\omega}_{k+1}\right]$, and $\phi_{k}$ is the argument of $\bar{\omega}_{k}-\bar{\omega}_{k+1}$ in $[-\pi / 2,3 \pi / 2)$. Let $e_{k}=\exp \left(i \phi_{k}\right)$. Certain $\bar{\omega}_{p}$ on $L_{k}$ are assigned doubly indexed subscripts as follows: Consider the convex hull of $\bar{\omega}_{k}, \bar{\omega}_{k+1}$ and the $\tau_{p}=\bar{\omega}_{p}+i m_{p} e_{k}$ for which $\bar{\omega}_{p}$ is on $L_{k}$; assign subscripts $j=1, \cdots, \sigma_{k}$ to $\omega_{k j}$ so that $\omega_{k 1}=\omega_{k}, \omega_{k \sigma_{k}}=\omega_{k+1}$, and $\tau_{k j}$ are vertices of this hull proceeding in a counterclockwise direction from $\bar{\omega}_{k}+i m_{k} e_{k}$ to $\bar{\omega}_{k+1}+i m_{k+1} e_{k}$. For $j=1, \cdots, \sigma_{k}-1, L_{k j}=\left[\tau_{k j}, \tau_{k j+1}\right]$, 
$\mu_{k j}$ is the quotient of $m_{k j}-m_{k j+1}$ by $\left(\omega_{k j}-\omega_{k j+1}\right) e_{k}$ and is real, and $n_{k j}$ is the number of $\tau_{p}$ on $L_{k j}$.

For $k=1, \cdots, \sigma, j=1, \cdots, \sigma_{k}-1$, and $H>0$, the set $V_{k j}(H)$ is the set

$$
\left\{z ; g\left(z / e_{k}\right) \geqq 0,\left|\Re\left(z / e_{k}\right)+\mu_{k j} \log \right| z|| \leqq H\right\} .
$$

Setting $z^{\prime}=z / e_{k}$, it is easy to show that for a fixed $H$ the subsets of the $V_{k j}(H)$ composed of $z$ of large modulus are individually connected and mutually disjoint with respect to pairs $(k, j)$. The boundary curves of $V_{k j}(H)$ are logarithmic to the exterior (to $Q$ ) normal to $L_{k}$ through the origin. For each $k$ and for each $\theta$ in $(0, \pi / 2), T_{k}(\theta)$ is a closed sector with vertex at the origin of opening $2 \theta$ about the outward normal to $L_{k}$ through the origin. For fixed $\theta$ and $H$, the subsets of $V_{k j}(H)$ composed of points of sufficiently large modulus are in $T_{k}(\theta)$.

For the same $k$ and $j$ and each triple of reals $(\alpha, s, H)$, where $s>0$ and $H>0$, the set $R_{k j}(\alpha, s, H)$ is given by

$$
\left\{z ; g\left(z / e_{k}\right)+\mu_{k j} \arg z \in[\alpha, \alpha+s],\left|\Re\left(z / e_{k}\right)+\mu_{k j} \log \right| z|| \leqq H\right\},
$$

where $\arg z \in\left(\phi_{k}, \phi_{k}+\pi\right)$. For fixed $\theta$ and $H, R_{k j}(\alpha, s, H)$ is in $V_{k j}(H) \cap T_{k}(\theta)$ for all $s>0$ if $\alpha$ is sufficiently large. If $\alpha$ is large, the set approximates a rectangle of dimensions $s$ by $2 H . N_{f}\left(R_{k j}(\alpha, s, H)\right)$ denotes the number of zeros of $f$ in closed $R_{k j}(\alpha, s, H)$, the zeros being counted according to their multiplicities.

For triples of reals $(\alpha, s, H)$ with $s>0$ and $H>0, R(\alpha, s, H)$, without subscripts, denotes the rectangle of points $z=x+i y$ with $|x| \leqq H$ and $y$ in $[\alpha, \alpha+s] . N_{o}(R(\alpha, s, H))$ denotes the number of zeros of the function $g$ in that rectangle.

3. Distribution of zeros. We first give Wilder's theorem, adapting Langer's proof so as to include all rectangles. In particular, the function in question may have zeros on the boundary of a rectangle.

ThEOREM 1. Let $g(z)=\sum_{j=1}^{n} A_{j} \exp \left(\omega_{j} z\right)$, where $z=x+i y, A_{j} \neq 0$, $\omega_{1}<\omega_{2}<\cdots<\omega_{n}$. Then there exists $K>0$ such that (a) each zero of $g$ is in $|x|<K$, and (b) for each pair of reals $(\alpha, s)$ with $s>0$,

$$
\left|N_{0}(R(\alpha, s, K))-s\left(\omega_{n}-\omega_{1}\right) /(2 \pi)\right| \leqq n-1 .
$$

Proof. Let $h(z)=g(z) \exp \left(-\omega_{1} z\right) / A_{1}$. Then $h$ and $g$ have the same zeros and $h(z)=1+\sum_{j=2}^{n} B_{j} \exp \left(\gamma_{j} z\right)$, where $\gamma_{j}=\omega_{j}-\omega_{1}, B_{j}=A_{j} / A_{1}$ and $0=\gamma_{1}<\cdots<\gamma_{n}$. For $x>0, h(z)=\left[1+\beta_{1}(z)\right] B_{n} \exp \left(\gamma_{n} z\right)$, where $\beta_{1}(z) \rightarrow 0$ as $x \rightarrow \infty$, while for $x<0, h(z)=1+\beta_{2}(z)$, where $\beta_{2}(z) \rightarrow 0$ as $x \rightarrow-\infty$. If $K$ is chosen so that $\left|\beta_{i}(z)\right|<1 / 2$ when $|x| \geqq K$, conclusion (a) follows. 
To establish (b) it suffices to show that for each $\epsilon>0$

$$
\left|N_{h}(R(\alpha, s, K))-s \gamma_{n} /(2 \pi)\right|<n-1+\epsilon .
$$

Given $\epsilon>0$, choose $H_{\epsilon} \geqq K$ so that $\left|\arg \left[1+\beta_{i}(z)\right]\right|<\pi \epsilon / 4$ respectively on the lines $x=(-1)^{i+1} H_{\epsilon}, i=1$, 2. For each $s>0$, choose $\delta=\delta(\epsilon, s)>0$ so that $\delta<\pi \epsilon /\left(2 \gamma_{n}\right), \delta<s / 2$, and so that $h$ has no zeros on the boundaries of

$$
R_{1}=R\left(\alpha-\delta, s+2 \delta, H_{\epsilon}\right)
$$

and

$$
R_{2}=R\left(\alpha+\delta, s-2 \delta, H_{\epsilon}\right) .
$$

This is possible since the zeros of $h$ are isolated. Consider changes in arg $h(z)$ as $z$ traverses $R_{1}$ in the positive direction. On the right boundary the change is at most $(s+2 \delta) \gamma_{n}+\pi \epsilon / 2$, while on the left it is at most $\pi \epsilon / 2$. On a line where $y$ is constant, $g(h(z))$ has the form $\sum_{j=2}^{n} E_{j} \exp \left(\gamma_{j} x\right)$ with $E_{j}$ real. By induction, such a function, if not identically zero, can have at most $n-2$ real zeros. Hence on a horizontal side of $R_{1}$, either $\mathscr{g}(h(z)) \equiv 0$ and the change in arg $h(z)$ is zero since $h$ has no zeros there, or the absolute value of the change is less than $(n-1) \pi$. Therefore the total change is bounded above by $(s+2 \delta) \gamma_{n}+\pi \epsilon+2 \pi(n-1)$. By our choice of $\delta$, it follows that $N_{h}(R(\alpha, s, K)) \leqq N_{h}\left(R_{1}\right)<n-1+\epsilon+s \gamma_{n} /(2 \pi)$. Similarly, $N_{h}(R(\alpha, s, K))$ $\geqq N_{h}\left(R_{2}\right)>-n+1-\epsilon+s \gamma_{n} /(2 \pi)$. The conclusion then follows.

We note that the conclusions of this theorem hold for each $H \geqq K$. That this is the best possible result involving all rectangles, i.e., all $\alpha$ and $s$, can be verified with the function $1+e^{z}$.

THEOREM 2. Given $f$ as in (1), then there exists $K>0$ such that (a) all but a finite number of zeros of $f$ of modulus greater than $r_{0}$ are in $\bigcup_{k, j} V_{k j}(K)$, and (b) for each pair of positive reals $\epsilon$ and $s_{0}$, there exists an $\alpha_{0}=\alpha_{0}\left(\epsilon, s_{0}\right)$ such that whenever $\alpha \geqq \alpha_{0}$ and $s \geqq s_{0}$,

$$
\left|N_{f}\left(R_{k j}(\alpha, s, K)\right)-s\right| \omega_{k j+1}-\omega_{k j}|/(2 \pi)|<n_{k j}-1+\epsilon .
$$

Before proving the theorem, we remark that the proofs of the theorems in $[1$, pp. 17, 21] that correspond to the theorems here, warrant only weak inequalities in their conclusions. In the second theorem there (in which the last sentence should be deleted), the magnitude of $s$ depends on $h$ as well as $H$. This is a deficiency for some applications and is not found in the more general theorem given here.

PROOF. Let $g_{k j}(z)=\sum A_{k p} \exp \left[\left(\omega_{k p}-\omega_{k j}\right) e_{k} z\right]$ where the sum is taken over $p$ for which $\tau_{k p} \in L_{k j}$. Choose $K>0$ sufficiently large so that the conclusion of Lemma 13 of [1] holds when $H=K$ (e.g., 
by insuring that $\left.\sum_{p \neq j}\left|A_{k p} / A_{k j}\right| \exp \left[-K\left|\omega_{k p}-\omega_{k j}\right|\right]<1 / 4\right)$ and so that Theorem 1 holds for $K$ with each $g_{k j}$. Part (a) then follows from Theorem 1 of [1] because of the first restriction on $K$.

Choose $r_{1}>0$ so that $r_{1} \geqq r_{0}$, all zeros of $f$ of modulus greater than $r_{1}$ are in $U_{k, j} V_{k j}(K)$, and so that the sets $V_{k j}(K+1) \cap\left\{z ;|z|>r_{1}\right\}$ are mutually disjoint with respect to pairs $(k, j)$. Let $\theta$ be fixed in $(0, \pi / 2)$, yielding sectors $T_{k}(\theta)$. Choose $\alpha_{1}>0$ sufficiently large so that if $\alpha \geqq \alpha_{1}$, then for all $s>0, R_{k j}(\alpha, s, K+1)$ is contained in

$$
\Omega=V_{k j}(K+1) \cap T_{k}(\theta) \cap\left\{z ;|z|>r_{1}\right\} .
$$

By Lemma 21 of [1], for all $z$ in $\Omega, f(z)$ is given by

$$
z^{m_{k j} e^{\omega k j z}} \sum A_{k p}[1+\epsilon(z)] \exp \left[e_{k}\left(\omega_{k p}-\omega_{k j}\right)\left(z / e_{k}+\mu_{k j} \log z\right)\right],
$$

where the sum is taken over all $p$ for which $\tau_{k p} \in L_{k j}$. The form of the $\epsilon(z)$ depends on $\theta$, and $\epsilon(z) \rightarrow 0$ as $z \rightarrow \infty$ in $\Omega$. The transformation $w=u+i v=z / e_{k}+\mu_{k j} \log z$, where $g(\log z) \in\left(\phi_{k}, \phi_{k}+\pi\right)$ maps $\Omega$ into the strip $|u| \leqq K+1$ with sets $R_{k j}(\alpha, s, K+\delta)$ mapping conformally onto rectangles $R(\alpha, s, K+\delta)$ in the $w$-plane when $\alpha \geqq \alpha_{1}, s>0$, and $0 \leqq \delta \leqq 1$.

Define $f_{k j}$ and $h_{k j}$ by $f(z)=f_{k j}(z) \exp \left[m_{k j} \log z+\omega_{k j} z\right]$ and $h_{k j}(w)$ $=f_{k j}(z)$, where $w=z / e_{k}+\mu_{k j} \log z$. It follows easily from (3) that $f_{k j}(z)=g_{k j}(w)+\epsilon(w)$ for $z$ in $\Omega$, where $\epsilon(w) \rightarrow 0$ as $w \rightarrow \infty$ in $|u| \leqq K+1$. Hence if $\alpha \geqq \alpha_{1}, s>0$, and $0 \leqq \delta \leqq 1$, then $N_{f}\left(R_{k j}(\alpha, s, K+\delta)\right)$ $=N_{f_{k j}}\left(R_{k j}(\alpha, s, K+\delta)\right)=N_{h_{k j}}(R(\alpha, s, K+\delta))$.

For given positive $\epsilon$ and $s_{0}$, choose $\delta=\delta\left(\epsilon, s_{0}\right)$ so that

$$
\delta<\pi \epsilon /\left|\omega_{k j+1}-\omega_{k j}\right|, \delta<s_{0} / 2 \text {, and } \delta<1 \text {. }
$$

Noting that the exponent coefficients in $g_{k j}$ are real, it follows from Theorem 1 that there is a bound on the number of zeros of $g_{k j}$ in $R(\alpha+s, \delta, K+\delta)$ and $R(\alpha-\delta, \delta, K+\delta)$ which is independent of $\alpha$ and $s$ but dependent on $\delta$ and hence on $\epsilon$ and $s_{0}$. As a result, in each of these rectangles one may pass a horizontal line that is uniformly (in $\alpha$ and $s$ ) bounded from the zeros of $g_{k j}$. For $s>0$ and $\alpha \geqq \alpha_{1}+s_{0} / 2$, let $R_{\alpha s}$ be a rectangle formed by such lines and the lines $u= \pm(K+\delta)$. Then $R_{\alpha s} \supset R(\alpha, s, K)$, and for $w$ on the boundary of $R_{\alpha s}, h_{k j}(w)=g_{k j}(w)\left[1+\epsilon(w) / g_{k j}(w)\right]$. When $w$ is on the boundary of the $R_{\alpha z}, w$ is uniformly bounded from the zeros of $g_{k j}$. By Theorem 3 of [1] it follows that $\left|g_{k j}(w)\right|$ is uniformly bounded from zero for such $w$. Choose $\alpha_{0}$ sufficiently large so that $\alpha_{0} \geqq \alpha_{1}$ $+s_{0} / 2$, and if $\alpha \geqq \alpha_{0}$ and $w$ is on the boundary of $R_{\alpha s}$, then $\left|\epsilon(w) / g_{k j}(w)\right|$ $<1$. Then for $\alpha \geqq \alpha_{0}$ and $s>0, h_{k j}$ and $g_{k j}$ have the same number of zeros in $R_{\alpha \theta}$. Using the second condition in the choice of $K$ 
and the fact that $\delta<\pi \epsilon /\left|\omega_{k j+1}-\omega_{k j}\right|$, for $\alpha \geqq \alpha_{0}$ and $s>0$, it follows that $N_{f}\left(R_{k j}(\alpha, s, K)\right)=N_{h_{k j}}(R(\alpha, s, K)) \leqq N_{h_{k j}}\left(R_{\alpha s}\right)=N_{g_{k j}}\left(R_{\alpha s}\right)$ $\leqq s\left|\omega_{k j+1}-\omega_{k j}\right| /(2 \pi)+n_{k j}-1+\epsilon$.

Since $\delta<s_{0} / 2$, when $s \geqq s_{0}$ one may construct rectangles with vertical sides on $u= \pm(K+\delta)$ and zero-free horizontal sides inside and within $\delta$ of those of $R(\alpha, s, K)$ on which $w$ is uniformly bounded from the zeros of $g_{k j}$. By our choice of $r_{1}$ and $\alpha_{1}, N_{f}\left(R_{k j}(\alpha, s, K)\right)$ $=N_{f}\left(R_{k j}(\alpha, s, K+\delta)\right)$ when $\alpha \geqq \alpha_{1}$ and $s>0$. An argument like the above (in which $\alpha_{0}$ may need to be increased) gives as a lower bound for $N_{f}\left(R_{k j}(\alpha, s, K)\right)$ the quantity $s\left|\omega_{k j+1}-\omega_{k j}\right| /(2 \pi)-n_{k j}+1-\epsilon$ if $s \geqq s_{0}$. Together, the two bounds establish (b).

If $\epsilon$ is chosen to be one, then for a fixed $s>0$ one can use the bound $n_{k j}$ in (b) for all large $\alpha$. If with $\epsilon=1 / 2$ one considers only $s$ for which $s\left|\omega_{k j+1}-\omega_{k j}\right| /(2 \pi)$ is a positive integer (such $s$ are automatically bounded from zero), then $n_{k j}-1$ serves as a weak bound in (b) for all large $\alpha$.

\section{REFERENCES}

1. D. G. Dickson, Expansions in series of solutions of linear difference-differential and infinite order differential equations with constant coefficients, Mem. Amer. Math. Soc. No. 23 (1957), 1-72.

2. - Asymptotic distribution of zeros of exponential sums, Publ. Math. Debrecen (to appear).

3. R. E. Langer, On the zeros of exponential sums and integrals, Bull. Amer. Math. Soc. 37 (1931), 213-239.

4. G. P6lya, Geometrisches über die Verteilung der Nullstellen gewisser ganzer transzendenter Funktionen, S.-B. Bayer. Akad., München (1920), 285-290.

5. - Untersuchungen ïber Lucken und Singularitäten von Potenzreihen, Math. Z. 29 (1929), 549-640.

6. E. Schwengeler, Geometrisches über die Verteilung der Nullstellen spezieller ganzer Funktionen, Dissertation, University of Zurich, Zurich, 1925.

7. J. D. Tamarkin, Some general problems of the theory of ordinary differential equations and expansion of an arbitrary function in series of fundamental functions, Math. Z. 27 (1928), 1-54.

8. C. E. Wilder, Expansion problems of ordinary linear differential equations with auxiliary conditions at more than two points, Trans. Amer. Math. Soc. 18 (1917), 415442.

The University of Michigan 